Commun. Korean Math. Soc. 29 (2014), No. 1, pp. 37-50

http://dx.doi.org/10.4134/CKMS.2014.29.1.037

\title{
ON A CLASS OF SEMILINEAR ELLIPTIC SYSTEMS INVOLVING GRUSHIN TYPE OPERATOR
}

\author{
Nguyen Thanh Chung
}

\begin{abstract}
Using variational methods, we prove some results on the nonexistence and multiplicity of weak solutions for a class of semilinear elliptic systems of two equations involving Grushin type operators with signchanging nonlinearities. We also shows that the similar results can be obtained for systems of $m$ equations, where $m$ is arbitrary.
\end{abstract}

\section{Introduction}

In recent years, more and more mathematicians have studied the existence of solutions for degenerate elliptic problems. This comes from the fact that they arise in many areas of applied physics, including nuclear physics, field theory, solid waves and problems of false vacuum. These problems are introduced as models for several physical phenomena related to equilibrium of continuous media which somewhere be perfect insulators (see $[8,19])$. However, the study have essentially based on the Caffarelli-Kohn-Nirenberg inequalities and their variants, see for example $[6,7,9,11,14,26]$ and the references therein. In this paper, we will study the existence of solutions for degenerate elliptic problems involving Grushin type operator $G_{s}=\Delta_{x}+|x|^{2 s} \Delta_{y}$ for $s \geq 0$. To our knowledge, the Grushin type operators were firstly introduced in [10], and developed in $[13,15,17,22,23,24,25]$.

Let $\Omega \subset \mathbb{R}^{N}=\mathbb{R}^{N_{1}} \times \mathbb{R}^{N_{2}}$ be a bounded domain with smooth boundary $\partial \Omega$, and $0 \in \Omega$. In this paper, we are interested in the semilinear elliptic system with Grushin type operator

$$
\left\{\begin{aligned}
L_{\alpha, \beta} w & =\lambda \nabla F & & \text { in } \Omega \\
w & =0 & & \text { on } \partial \Omega
\end{aligned}\right.
$$

where

$$
w=(u, v), \quad L_{\alpha, \beta}=\left(\begin{array}{cc}
-G_{\alpha} & 0 \\
0 & -G_{\beta}
\end{array}\right), \quad G_{s}=\Delta_{x}+|x|^{2 s} \Delta_{y} \text { for } s \geq 0,
$$

Received July 4, 2012; Revised June 17, 2013.

2010 Mathematics Subject Classification. 34B27, 35J60, 35B05.

Key words and phrases. semilinear elliptic systems, Grushin type operator, non-existence, multiplicity, variational methods. 


$$
\Delta_{x}=\sum_{i=1}^{N_{1}} \frac{\partial^{2}}{\partial x_{i}^{2}}, \quad \Delta_{y}=\sum_{j=1}^{N_{2}} \frac{\partial^{2}}{\partial y_{j}^{2}},
$$

and $\nabla F=\left(F_{u}, F_{v}\right)$ stands for the gradient of a $C^{1}$ function $F: \Omega \times \mathbb{R} \times \mathbb{R} \rightarrow \mathbb{R}$ which may change sign, $\alpha, \beta \geq 0, \lambda$ is a positive parameter. Denoting $N(s)=$ $N_{1}+(s+1) N_{2}$, we assume that $N_{1}, N_{2} \geq 1$ and $N(\alpha)>2$ and $N(\beta)>2$.

We point the fact that in [5], N. M. Chuong et al. studied the existence of at least a weak solutions for problem (1.1), using the mountain pass theorem combined with the Ekeland principle. They also observed on the behaviour of the solutions when the parameter $\lambda \rightarrow 0$. The goal of this note is to give some sufficient conditions on the nonlinear terms to get the non-existence and multiplicity of weak solutions for (1.1). Thus, the results introduced here extend or complement the obtained results in [5]. Moreover, we do not require in this paper the Ambrosetti-Rabinowitz type condition as in [5]. We also shows that similar arguments can be applied to the systems of $m$ equations, where $m$ is arbitrary. Our paper is inspired by the ideas introduced in $[2,3,20]$, in which the authors considered the problem with the $p$-Laplace operator $-\Delta_{p}$. Regarding systems of Hamiltonian form involving Grushin type operators, the reader may find in the papers $[4,12]$.

Throughout this paper for $(t, s) \in \mathbb{R}^{2}$, we denote $|(t, s)|^{2}=|t|^{2}+|s|^{2}$. We assume that $F: \Omega \times \mathbb{R} \times \mathbb{R} \rightarrow \mathbb{R}$ is a $C^{1}$-function, satisfying the following conditions:

(f1) $F(x, y, 0,0)=0$ for a.e. $(x, y) \in \Omega, F(x, y, t, s)=F(x, y, 0, s)$ for all $t \leq 0, s \in \mathbb{R}$ and a.e. $(x, y) \in \Omega, F(x, y, t, s)=F(x, y, t, 0)$ for all $t \in \mathbb{R}, s \leq 0$ and a.e. $(x, y) \in \Omega$;

(f2) There exists a constant $C>0$ such that

$$
\left|F_{t}(x, y, t, s)\right|+\left|F_{s}(x, y, t, s)\right| \leq C(1+|t|+|s|)
$$

for all $t, s \in \mathbb{R}$ and a.e. $(x, y) \in \Omega$.

We say that a function $\gamma$ verifies the property $(\Gamma)$ if and only if

$$
\gamma(t, s) \leq M\left(|t|^{2}+|s|^{2}\right)
$$

for all $t, s \in \mathbb{R}$, where $M>0$ is independent of $\gamma$. Let $H_{i}, i=1,2$ be two functions satisfying property $\Gamma$. Motivated by an eigenvalue problem considered in [2], we introduce the following assumptions on the behavior of $F$ at the origin and at infinity:

(f3) It holds that

$$
\limsup _{|(t, s)| \rightarrow 0} \frac{F(x, y, t, s)}{H_{1}(t, s)} \leq 0
$$

uniformly in $(x, y) \in \Omega$;

(f4) It holds that

$$
\limsup _{|(t, s)| \rightarrow \infty} \frac{F(x, y, t, s)}{H_{2}(t, s)} \leq 0
$$


uniformly in $(x, y) \in \Omega$.

We denote by $S^{p, s}(\Omega), 1 \leq p<\infty$ the set of element $u \in L^{p}(\Omega)$ such that $\frac{\partial u}{\partial x_{i}},|x|^{s} \frac{\partial u}{\partial y_{j}} \in L^{p}(\Omega)$ for all $i=1,2, \ldots, N_{1}$, and $j=1,2, \ldots, N_{2}$. Then $S^{p, s}(\Omega)$ is a Banach space with respect to the norm

$$
\|u\|_{p, s}=\left[\int_{\Omega}\left(|u|^{p}+\left|\nabla_{x} u\right|^{p}+|x|^{p s}\left|\nabla_{y} u\right|^{p}\right) d x d y\right]^{\frac{1}{p}}
$$

where

$$
\nabla_{x}=\left(\frac{\partial}{\partial x_{1}}, \frac{\partial}{\partial x_{2}}, \ldots, \frac{\partial}{\partial x_{N_{1}}}\right), \quad \nabla_{y}=\left(\frac{\partial}{\partial y_{1}}, \frac{\partial}{\partial y_{2}}, \ldots, \frac{\partial}{\partial y_{N_{2}}}\right) .
$$

The space $S_{0}^{p, s}(\Omega)$ is the closure of $C_{0}^{1}(\Omega)$ in the space $S^{p, s}(\Omega)$. In the special case when $p=2$, we conclude the Hilbert spaces $S^{2, s}(\Omega)$ and $S_{0}^{2, s}(\Omega)$. In [22], the authors proved that for all $u \in S_{0}^{2, s}(\Omega)$,

$$
\left(\int_{\Omega}|u|^{q} d x d y\right)^{\frac{1}{q}} \leq C\left[\int_{\Omega}\left(\left|\nabla_{x} u\right|^{2}+|x|^{2 s}\left|\nabla_{y} u\right|^{2}\right) d x d y\right]^{\frac{1}{2}},
$$

where $q=\frac{2 N(s)}{N(s)-2}-\tau, C>0, s \geq 0$ and $N(s)=N_{1}+(s+1) N_{2}$, provided that $\tau>0$ is small enough. Furthermore, the embedding $S_{0}^{2, s}(\Omega)$ into $L^{q}(\Omega)$ is compact for $2 \leq q<2_{s}^{*}=\frac{2 N(s)}{N(s)-2}$. Therefore, in the space $S_{0}^{2, s}(\Omega)$, we can use the following equivalent norm

$$
\|u\|_{2, s}=\left[\int_{\Omega}\left(\left|\nabla_{x} u\right|^{2}+|x|^{2 s}\left|\nabla_{y} u\right|^{2}\right) d x d y\right]^{\frac{1}{2}} .
$$

Next, for $\alpha, \beta \geq 0$, we define the space $H=S_{0}^{2, \alpha}(\Omega) \times S_{0}^{2, \beta}(\Omega)$. Then $H$ is a Hilbert space under the norm

$$
\|w\|=\|u\|_{2, \alpha}+\|v\|_{2, \beta}
$$

and the inner product is

$\langle u, v\rangle_{H}=\int_{\Omega}\left(\nabla_{x} u_{1} \cdot \nabla_{x} v_{1}+\nabla_{x} u_{2} \cdot \nabla_{x} v_{2}+|x|^{2 \alpha} \nabla_{y} u_{1} \cdot \nabla_{y} v_{1}+|x|^{2 \beta} \nabla_{y} u_{2} \cdot \nabla_{y} v_{2}\right) d x d y$ for all $u=\left(u_{1}, v_{1}\right), v=\left(v_{1}, v_{2}\right) \in H$.

Definition 1.1. We say that a function $w=(u, v) \in H$ is a weak solution of system (1.1) if and only if

$$
\begin{aligned}
& \int_{\Omega}\left(\nabla_{x} u \cdot \nabla_{x} \varphi_{1}+|x|^{2 \alpha} \nabla_{y} u \cdot \nabla_{y} \varphi_{1}\right) d x d y=\lambda \int_{\Omega} F_{u}(x, y, u, v) \varphi_{1} d x d y \\
& \int_{\Omega}\left(\nabla_{x} v \cdot \nabla_{x} \varphi_{2}+|x|^{2 \beta} \nabla_{y} v \cdot \nabla_{y} \varphi_{2}\right) d x d y=\lambda \int_{\Omega} F_{v}(x, y, u, v) \varphi_{2} d x d y
\end{aligned}
$$

for all $\varphi=\left(\varphi_{1}, \varphi_{2}\right) \in H$.

The main results of this paper can be described in the following theorems. 
Theorem 1.2. Assume $F: \Omega \times \mathbb{R} \times \mathbb{R} \rightarrow \mathbb{R}$, satisfies

$$
\left|F_{t}(x, y, t, s)\right|+\left|F_{s}(x, y, t, s)\right| \leq C(|t|+|s|), \quad C>0
$$

for all $(t, s) \in \mathbb{R}^{2}$ and a.e. $(x, y) \in \Omega$. Then there is a constant $\lambda_{*}>0$ such that system (1.1) has no nontrivial weak solution for any $\lambda<\lambda_{*}$.

Theorem 1.3. Assume that the conditions (f1)-(f4) are satisfied. Moreover, if in addition we assume that there exist a ball $B \subset \Omega$ and $t_{0}, s_{0}>0$ such that $F\left(x, y, t_{0}, s_{0}\right)>0$ for all $(x, y) \in B$, then there exists a constant $\lambda^{*}>0$ such that system (1.1) has at least two nontrivial, nonnegative weak solutions for any $\lambda \geq \lambda^{*}$.

Our paper is organized as follows. In Section 2, we prove Theorems 1.2 and 1.3 using variational arguments. In Section 3, we make some comments regarding extensions of system (1.1).

\section{Proofs of the main results}

Proof of Theorem 1.2. Let $\lambda_{1, \alpha}, \lambda_{1, \beta}$ be the first eigenvalue of the operators $-G_{\alpha}$ and $-G_{\beta}$ with Dirichlet boundary, i.e.,

$$
\begin{aligned}
& \lambda_{1, \alpha}=\inf _{u \in S_{0}^{2, \alpha}(\Omega) \backslash\{0\}} \frac{\int_{\Omega}\left(\left|\nabla_{x} u\right|^{2}+|x|^{2 \alpha}\left|\nabla_{y} u\right|^{2}\right) d x d y}{\int_{\Omega}|u|^{2} d x d y}>0, \\
& \lambda_{1, \beta}=\inf _{v \in S_{0}^{2, \beta}(\Omega) \backslash\{0\}} \frac{\int_{\Omega}\left(\left|\nabla_{x} v\right|^{2}+|x|^{2 \beta}\left|\nabla_{y} v\right|^{2}\right) d x d y}{\int_{\Omega}|v|^{2} d x d y}>0 .
\end{aligned}
$$

Then we have

$$
\begin{aligned}
0<\lambda_{\alpha, \beta} & =\min \left\{\lambda_{1, \alpha}, \lambda_{1, \beta}\right\} \\
& \leq \frac{\int_{\Omega}\left[\left|\nabla_{x} u\right|^{2}+\left|\nabla_{x} v\right|^{2}+|x|^{2 \alpha}\left|\nabla_{y} u\right|^{2}+|x|^{2 \beta}\left|\nabla_{y} v\right|^{2}\right] d x d y}{\int_{\Omega}\left[|u|^{2}+|v|^{2}\right] d x d y}
\end{aligned}
$$

for all $w=(u, v) \in H \backslash\{0\}$ (see [22]).

If $w=(u, v) \in H$ is a non-trivial weak solution of system (1.1), it follows by multiplying the first equation of (1.1) by $u$ and the second by $v$, and integrating by parts that

$$
\begin{aligned}
& \int_{\Omega}\left(\left|\nabla_{x} u\right|^{2}+|x|^{2 \alpha}\left|\nabla_{y} u\right|^{2}\right) d x=\lambda \int_{\Omega} F_{u}(x, y, u, v) u d x d y, \\
& \int_{\Omega}\left(\left|\nabla_{x} v\right|^{2}+|x|^{2 \beta}\left|\nabla_{y} v\right|^{2}\right) d x=\lambda \int_{\Omega} F_{v}(x, y, u, v) v d x d y,
\end{aligned}
$$


which implies by using (1.3) that

$$
\begin{aligned}
& \int_{\Omega}\left(\left|\nabla_{x} u\right|^{2}+\left|\nabla_{x} v\right|^{2}+|x|^{2 \alpha}\left|\nabla_{y} u\right|^{2}+|x|^{2 \beta}\left|\nabla_{y} v\right|^{2}\right) d x d y \\
= & \lambda \int_{\Omega}\left(F_{u}(x, y, u, v) u+F_{v}(x, y, u, v) v\right) d x d y \\
\leq & \lambda C \int_{\Omega}\left(|u|^{2}+|v|^{2}\right) d x d y,
\end{aligned}
$$

where $C$ is a positive constant. Thus, by taking $\lambda_{*}=\frac{\lambda_{\alpha, \beta}}{C}>0$, where $\lambda_{\alpha, \beta}$ is given by (2.4), we conclude the proof of Theorem 1.2.

In order to prove Theorem 1.3, we shall use critical point theory. For all $\mu, \lambda \in \mathbb{R}$, we consider the functional $T_{\lambda}: H \rightarrow \mathbb{R}$ given by

$$
\begin{aligned}
T_{\lambda}(w)= & \frac{1}{2} \int_{\Omega}\left(\left|\nabla_{x} u\right|^{2}+\left|\nabla_{x} v\right|^{2}+|x|^{2 \alpha}\left|\nabla_{y} u\right|^{2}+|x|^{2 \beta}\left|\nabla_{y} v\right|^{2}\right) d x d y \\
& -\lambda \int_{\Omega} F(x, y, u, v) d x d y \\
= & J(w)-\lambda I(w), \quad w=(u, v) \in H,
\end{aligned}
$$

where

$$
\begin{aligned}
& J(w)=\frac{1}{2} \int_{\Omega}\left(\left|\nabla_{x} u\right|^{2}+\left|\nabla_{x} v\right|^{2}+|x|^{2 \alpha}\left|\nabla_{y} u\right|^{2}+|x|^{2 \beta}\left|\nabla_{y} v\right|^{2}\right) d x d y, \\
& I(w)=\int_{\Omega} F(x, y, u, v) d x d y, \quad w=(u, v) \in H .
\end{aligned}
$$

A simple computation implies that $T_{\lambda}$ is well-defined and of $C^{1}$ class in $H$. Thus, weak solutions of system (1.1) correspond to the critical points of $T_{\lambda}$. Moreover, we first have the following result.

Lemma 2.1. The functional $T_{\lambda}$ given by (2.6) is weakly semi-continuous in $H$.

Proof. Let $\left\{w_{m}\right\}=\left\{\left(u_{m}, v_{m}\right)\right\}$ be a sequence that converges weakly to $w=$ $(u, v)$ in $H$. By the semi-continuity of norm, it is sufficient to show that the functional $I$ is weakly continuous in $H$, i.e.,

$$
\lim _{m \rightarrow \infty} \int_{\Omega} F\left(x, y, u_{m}, v_{m}\right) d x d y=\int_{\Omega} F(x, y, u, v) d x d y .
$$

Indeed, we have

$$
\begin{aligned}
& \int_{\Omega}\left[F\left(x, y, u_{m}, v_{m}\right)-F(x, y, u, v)\right] d x d y \\
= & \int_{\Omega} \nabla F\left(x, y, w+\theta_{m}\left(w_{m}-w\right)\right) \cdot\left(w_{m}-w\right) d x d y \\
= & \int_{\Omega} F_{u}\left(x, y, u+\theta_{1, m}\left(u_{m}-u\right), v+\theta_{2, m}\left(v_{m}-v\right)\right)\left(u_{m}-u\right) d x d y
\end{aligned}
$$




$$
+\int_{\Omega} F_{v}\left(x, y, u+\theta_{1, m}\left(u_{m}-u\right), v+\theta_{2, m}\left(v_{m}-v\right)\right)\left(v_{m}-v\right) d x d y,
$$

where $\theta_{m}=\left(\theta_{1, m}, \theta_{2, m}\right)$ and $0 \leq \theta_{1, m}(x), \theta_{2, m}(x) \leq 1$ for all $x \in \Omega$. Now, using (f2) and Hölder's inequality we conclude that

$$
\begin{aligned}
& \left|\int_{\Omega}\left[F\left(x, y, u_{m}, v_{m}\right)-F(x, y, u, v)\right] d x d y\right| \\
\leq & \int_{\Omega}\left|F_{u}\left(x, y, u+\theta_{1, m}\left(u_{m}-u\right), v+\theta_{2, m}\left(v_{m}-v\right)\right)\right|\left|u_{m}-u\right| d x d y \\
& +\int_{\Omega}\left|F_{v}\left(x, y, u+\theta_{1, m}\left(u_{m}-u\right), v+\theta_{2, m}\left(v_{m}-v\right)\right)\right|\left|v_{m}-v\right| d x d y \\
\leq & \left.C \int_{\Omega}\left(1+\left|u+\theta_{1, m}\left(u_{m}-u\right)\right|+\mid v+\theta_{2, m}\left(v_{m}-v\right)\right) \mid\right) \\
& \left(\left|u_{m}-u\right|+\left|v_{m}-v\right|\right) d x d y \\
\leq & C\left(|\Omega|^{\frac{1}{2}}+\left\|u+\theta_{1, m}\left(u_{m}-u\right)\right\|_{L^{2}}+\left\|v+\theta_{1, m}\left(v_{m}-v\right)\right\|_{L^{2}}\right) \\
& \left(\left\|u_{m}-u\right\|_{L^{2}}+\left\|v_{m}-v\right\|_{L^{2}}\right) .
\end{aligned}
$$

On the other hand, since $H \hookrightarrow L^{2}(\Omega) \times L^{2}(\Omega)$ is compact, the sequence $\left\{w_{m}\right\}$ converges strongly to $w=(u, v)$ in the space $L^{2}(\Omega) \times L^{2}(\Omega)$, i.e., $\left\{u_{m}\right\}$ converges strongly to $u$ in $L^{2}(\Omega)$ and $\left\{v_{m}\right\}$ converges strongly to $v$ in $L^{2}(\Omega)$. Hence, it is easy to see that the sequences $\left\{\left\|u+\theta_{1, m}\left(u_{m}-u\right)\right\|_{L^{2}}\right\}$ and $\left\{\left\|v+\theta_{2, m}\left(v_{m}-v\right)\right\|_{L^{2}}\right\}$ are bounded. Thus, it follows that (2.8) holds true.

Lemma 2.2. For any $\lambda \in \mathbb{R}$, the functional $T_{\lambda}$ is coercive and bounded from below on $H$.

Proof. For any $\lambda \in \mathbb{R}$, by conditions (f2) and (f4), there exists a constant $C_{\lambda}=C(\lambda)>0$ such that

$$
\lambda F(x, y, t, s) \leq \frac{\lambda_{\alpha, \beta}}{4 M} H_{2}(t, s)+C_{\lambda}
$$

for all $t, s \in \mathbb{R}$ and a.e. $x \in \Omega$. Hence, we have for all $w=(u, v) \in H$ that

$$
\begin{aligned}
T_{\lambda}(w)= & \frac{1}{2} \int_{\Omega}\left(\left|\nabla_{x} u\right|^{2}+\left|\nabla_{x} v\right|^{2}+|x|^{2 \alpha}\left|\nabla_{y} u\right|^{2}+|x|^{2 \beta}\left|\nabla_{y} v\right|^{2}\right) d x d y \\
& -\lambda \int_{\Omega} F(x, y, u, v) d x d y \\
\geq & \frac{1}{2}\|u\|_{2, \alpha}^{2}+\frac{1}{2}\|v\|_{2, \beta}^{2}-\int_{\Omega}\left(\frac{\lambda_{\alpha, \beta}}{4 M} H_{2}(u, v)+C_{\lambda}\right) d x d y \\
\geq & \frac{1}{4}\|u\|_{2, \alpha}^{2}+\frac{1}{4}\|v\|_{2, \beta}^{2}-C_{\lambda}|\Omega|
\end{aligned}
$$

which helps us to show that $T_{\lambda}$ is coercive and bounded from below in $H$. 
Lemma 2.3. If $w=(u, v) \in H$ is a weak solution of system (1.1), then $u \geq 0$ and $v \geq 0$ in $\Omega$.

Proof. Indeed, if $w=(u, v) \in H$ is a weak solution of system (1.1), then we have

$$
\begin{aligned}
0= & T_{\lambda}^{\prime}(w)\left(w^{-}\right) \\
= & \int_{\Omega}\left(\nabla u_{x} \cdot \nabla_{x} u^{-}+\nabla v_{x} \cdot \nabla_{x} v^{-}+|x|^{2 \alpha} \nabla u_{y} \cdot \nabla_{y} u^{-}+|x|^{2 \alpha} \nabla v_{y} \cdot \nabla_{y} v^{-}\right) d x d y \\
& \quad-\lambda \int_{\Omega}\left(F_{u}(x, y, u, v) u^{-}+F_{v}(x, y, u, v) v^{-}\right) d x d y \\
= & \int_{\Omega}\left(\left|\nabla_{x} u^{-}\right|^{2}+\left|\nabla_{x} v^{-}\right|^{2}+|x|^{2 \alpha}\left|\nabla_{y} u^{-}\right|^{2}+|x|^{2 \alpha}\left|\nabla_{y} v^{-}\right|^{2}\right) d x d y,
\end{aligned}
$$

where $w^{-}=\left(u^{-}, v^{-}\right)$with $u^{-}=\min \{0, u(x)\}$ is the negative part of $u$ and $v^{-}=\min \{0, v(x)\}$ is the negative part of $v$. Moreover, since

$$
0=\left\|u^{-}\right\|_{2, \alpha}^{2}+\left\|v^{-}\right\|_{2, \beta}^{2} \geq \lambda_{\alpha, \beta} \int_{\Omega}\left(\left|u^{-}\right|^{2}+\left|v^{-}\right|^{2}\right) d x d y
$$

it follows that $u(x) \geq 0$ and $v(x) \geq 0$ for a.e. $x \in \Omega$, i.e., $w \geq 0$ for a.e. $x \in \Omega$.

Lemma 2.4. There exists $\lambda^{*}>0$ such that $\inf _{H} T_{\lambda}<0$ for any $\lambda \geq \lambda^{*}$.

Proof. Let us consider a sufficiently large compact subset $B^{\prime}$ of $B$, where $B$ is a ball such that $F\left(x, y, t_{0}, s_{0}\right)>0$ for all $(x, y) \in B$ and some $t_{0}, s_{0}>0$. Consider $u_{0}$ and $v_{0}$, smooth functions with compact support in $B$, such that $u_{0}(x, y)=t_{0}, v_{0}(x, y)=s_{0}$ in $B^{\prime}, 0 \leq u_{0}(x, y) \leq t_{0}$ and $0 \leq v_{0}(x, y) \leq s_{0}$ for all $(x, y) \in B \backslash B^{\prime}$. Then we get

$$
\begin{aligned}
\int_{\Omega} F\left(x, y, u_{0}, v_{0}\right) d x d y & =\int_{B^{\prime}} F\left(x, y, u_{0}, v_{0}\right) d x d y+\int_{B \backslash B^{\prime}} F\left(x, y, u_{0}, v_{0}\right) d x d y \\
& \geq \int_{B^{\prime}} F\left(x, y, t_{0}, s_{0}\right) d x d y-C \int_{B \backslash B^{\prime}}\left(\left|u_{0}\right|^{2}+\left|v_{0}\right|^{2}\right) d x d y \\
& \geq \int_{B^{\prime}} F\left(x, y, t_{0}, s_{0}\right) d x d y-C\left(1+\left|t_{0}\right|^{2}+\left|s_{0}\right|^{2}\right)\left|B \backslash B^{\prime}\right| \\
& >0
\end{aligned}
$$

provided that $\left|B \backslash B^{\prime}\right|$ is small enough. Hence, denoting $w_{0}=\left(u_{0}, v_{0}\right) \in H$, we have

$$
\begin{aligned}
T_{\lambda}\left(w_{0}\right)= & \frac{1}{2} \int_{\Omega}\left(\left|\nabla_{x} u_{0}\right|^{2}+\left|\nabla_{x} v_{0}\right|^{2}+|x|^{2 \alpha}\left|\nabla_{y} u_{0}\right|^{2}+|x|^{2 \beta}\left|\nabla_{y} v_{0}\right|^{2}\right) d x d y \\
& -\lambda \int_{\Omega} F\left(x, y, u_{0}, v_{0}\right) d x d y \\
\leq & \frac{1}{2}\left\|u_{0}\right\|_{2, \alpha}^{2}+\frac{1}{2}\left\|v_{0}\right\|_{2, \beta}^{2}-\lambda \int_{\Omega} F\left(x, y, u_{0}, v_{0}\right) d x d y<0
\end{aligned}
$$


for $\lambda$ large enough. So there is a positive constant $\lambda^{*}$ such that $\inf _{H} T_{\lambda}<0$ for all $\lambda \geq \lambda^{*}$.

Now, we fix $\lambda \geq \lambda^{*}$. Then by Lemmas 2.1-2.4 the functional $T_{\lambda}$ has a global minimum $w_{1}=\left(u_{1}, v_{1}\right)$ such that $T_{\lambda}\left(w_{1}\right)<0$ and thus system (1.1) has a non-negative nontrivial weak solution $w_{1}=\left(u_{1}, v_{1}\right) \in H$. In order to obtain the second one, we use the mountain pass theorem [1]. To this purpose, we first show that $T_{\lambda}$ has the mountain pass geometry.

Lemma 2.5. For any $\lambda \in \mathbb{R}$, there exist $\rho \in\left(0,\left\|w_{1}\right\|_{H}\right)$ and a constant $r>0$ such that $T_{\lambda}(w) \geq r$ for all $w=(u, v) \in H$ with $\|w\|_{H}=\rho$.

Proof. For any $\lambda \in \mathbb{R}$, it follows from (f2) and (f3) that there is $C_{\lambda}>0$, which depends on $\lambda$, such that

$$
\lambda F(x, y, t, s) \leq \frac{\lambda_{\alpha, \beta}}{4 M} H_{1}(t, s)+C_{\lambda}\left(|t|^{p}+|s|^{q}\right)
$$

for all $(x, y, t, s) \in \Omega \times \mathbb{R} \times \mathbb{R}$, where $2<p<2_{\alpha}^{*}=\frac{2 N(\alpha)}{N(\alpha)-2}, 2<q<2_{\beta}^{*}=\frac{2 N(\beta)}{N(\beta)-2}$. Hence, by the Sobolev embeddings, it follows that

$$
\begin{aligned}
T_{\lambda}(w)= & \frac{1}{2} \int_{\Omega}\left(\left|\nabla_{x} u\right|^{2}+\left|\nabla_{x} v\right|^{2}+|x|^{2 \alpha}\left|\nabla_{y} u\right|^{2}+|x|^{2 \beta}\left|\nabla_{y} v\right|^{2}\right) d x d y \\
& -\lambda \int_{\Omega} F(x, y, u, v) d x d y \\
\geq & \frac{1}{2}\|u\|_{2, \alpha}^{2}+\frac{1}{2}\|v\|_{2, \beta}^{2}-\frac{\lambda_{\alpha, \beta}}{4 M} \int_{\Omega} H_{1}(u, v) d x d y-C_{\lambda} \int_{\Omega}\left(|u|^{p}+|v|^{q}\right) d x d y \\
\geq & \left(\frac{1}{4}-\bar{C}_{\lambda}\|u\|_{2, \alpha}^{p-2}\right)\|u\|_{2, \alpha}^{2}+\left(\frac{1}{4}-\bar{C}_{\lambda}\|v\|_{2, \beta}^{q-2}\right)\|v\|_{2, \beta}^{2},
\end{aligned}
$$

where $\bar{C}_{\lambda}$ is a positive constant. Since $2<p<2_{\alpha}^{*}=\frac{2 N(\alpha)}{N(\alpha)-2}, 2<q<2_{\beta}^{*}=$ $\frac{2 N(\beta)}{N(\beta)-2}$, there are two constants $r>0$ and $0<\rho<\left\|w_{1}\right\|_{H}$ such that $T_{\lambda}(w) \geq r$ for all $w \in H$ with $\|w\|_{H}=\rho$.

Lemma 2.6. The functional $T_{\lambda}$ satisfies the Palais-Smale condition in $H$.

Proof. Let $\left\{w_{m}\right\}=\left\{\left(u_{m}, v_{m}\right)\right\}$ be a Palais-Smale sequence for the functional $T_{\lambda}$ in $H$, i.e.,

$$
\left|T_{\lambda}\left(w_{m}\right)\right| \leq \bar{c} \text { for all } m, \quad T_{\lambda}^{\prime}\left(w_{m}\right) \rightarrow 0 \text { in } H^{-1} \text { as } m \rightarrow \infty,
$$

where $H^{-1}$ is the dual space of $H$.

Since $T_{\lambda}$ is coercive on $H$, the sequence $\left\{w_{m}\right\}$ is bounded in $H$. Since $H$ is a Hilbert space, there exists $w=(u, v) \in H$ such that, passing to a subsequence, still denoted by $\left\{w_{m}\right\}=\left\{\left(u_{m}, v_{m}\right)\right\}$, it converges weakly to $w=(u, v)$ in $H$ and hence $\left\{w_{m}\right\}$ converges strongly to $w$ in $L^{2}\left(\Omega, \mathbb{R}^{2}\right)$, i.e., $\left\{u_{m}\right\}$ converges 
strongly to $u$ in $L^{2}(\Omega)$ and $\left\{v_{m}\right\}$ converges strongly to $v$ in $L^{2}(\Omega)$. By the definition of $T_{\lambda}$ we have

$$
\begin{aligned}
& T_{\lambda}^{\prime}\left(w_{m}\right)\left(w_{m}-w\right) \\
= & \int_{\Omega}\left(\nabla_{x} u_{m} \cdot\left(\nabla_{x} u_{m}-\nabla_{x} u\right)+\nabla_{x} v_{m} \cdot\left(\nabla_{x} v_{m}-\nabla_{x} v\right)\right) d x d y \\
& +\int_{\Omega}\left(|x|^{2 \alpha} \nabla_{y} u_{m} \cdot\left(\nabla_{y} u_{m}-\nabla_{y} u\right)+|x|^{2 \beta} \nabla_{y} v_{m} \cdot\left(\nabla_{y} v_{m}-\nabla_{y} v\right)\right) d x d y \\
& -\lambda \int_{\Omega}\left(F_{u}\left(x, y, u_{m}, v_{m}\right)\left(u_{m}-u\right)+F_{v}\left(x, y, u_{m}, v_{m}\right)\left(v_{m}-v\right)\right) d x d y
\end{aligned}
$$

and

$$
\begin{aligned}
& T_{\lambda}^{\prime}(w)\left(w-w_{m}\right) \\
= & \int_{\Omega}\left(\nabla_{x} u \cdot\left(\nabla_{x} u-\nabla_{x} u_{m}\right)+\nabla_{x} v \cdot\left(\nabla_{x} v-\nabla_{x} v_{m}\right)\right) d x d y \\
& +\int_{\Omega}\left(|x|^{2 \alpha} \nabla_{y} u \cdot\left(\nabla_{y} u-\nabla_{y} u_{m}\right)+|x|^{2 \beta} \nabla_{y} v \cdot\left(\nabla_{y} v-\nabla_{y} v_{m}\right)\right) d x d y \\
& -\lambda \int_{\Omega}\left(F_{u}(x, y, u, v)\left(u-u_{m}\right)+F_{v}(x, y, u, v)\left(v-v_{m}\right)\right) d x d y .
\end{aligned}
$$

By (2.12) and (2.13) we get

$$
\begin{aligned}
& T_{\lambda}^{\prime}\left(w_{m}\right)\left(w_{m}-w\right)+T_{\lambda}^{\prime}(w)\left(w-w_{m}\right) \\
= & \int_{\Omega}\left(\left|\nabla_{x} u_{m}-\nabla_{x} u\right|^{2}+\left|\nabla_{x} v_{m}-\nabla_{x} v\right|^{2}\right) d x d y \\
& +\int_{\Omega}\left(|x|^{2 \alpha}\left|\nabla_{y} u_{m}-\nabla_{y} u\right|^{2}+|x|^{2 \beta}\left|\nabla_{y} v_{m}-\nabla_{y} v\right|^{2}\right) d x d y \\
& -\lambda \int_{\Omega}\left(F_{u}\left(x, y, u_{m}, v_{m}\right)-F_{u}(x, y, u, v)\right)\left(u_{m}-u\right) d x d y \\
& -\lambda \int_{\Omega}\left(F_{v}\left(x, y, u_{m}, v_{m}\right)-F_{v}(x, y, u, v)\right)\left(v_{m}-v\right) d x d y .
\end{aligned}
$$

By (2.11), we get

$$
\lim _{m \rightarrow \infty} T_{\mu, \lambda}^{\prime}\left(w_{m}\right)\left(w_{m}-w\right)+T_{\mu, \lambda}^{\prime}(w)\left(w-w_{m}\right)=0 .
$$

On the other hand, by using the condition (f2), Hölder's inequality and the compact embeddings we deduce that

$$
\begin{aligned}
& \left|\int_{\Omega}\left(F_{u}\left(x, y, u_{m}, v_{m}\right)-F_{u}(x, y, u, v)\right)\left(u_{m}-u\right) d x d y\right| \\
\leq & C \int_{\Omega}\left(2+\left|u_{m}\right|+\left|v_{m}\right|+|u|+|v|\right)\left|u_{m}-u\right| d x d y
\end{aligned}
$$




$$
\leq C\left(2|\Omega|^{\frac{1}{2}}+\left\|u_{m}\right\|_{L^{2}}+\left\|v_{m}\right\|_{L^{2}}^{2}+\|u\|_{L^{2}}+\|v\|_{L^{2}}^{2}\right)\left\|u_{m}-u\right\|_{L^{2}}^{2},
$$

which tends 0 as $m \rightarrow \infty$ and

$$
\begin{aligned}
& \left|\int_{\Omega}\left(F_{v}\left(x, y, u_{m}, v_{m}\right)-F_{v}(x, y, u, v)\right)\left(v_{m}-v\right) d x d y\right| \\
\leq & C \int_{\Omega}\left(2+\left|u_{m}\right|+\left|v_{m}\right|+|u|+|v|\right)\left|v_{m}-v\right| d x d y \\
\leq & C\left(2|\Omega|^{\frac{1}{2}}+\left\|u_{m}\right\|_{L^{2}}+\left\|v_{m}\right\|_{L^{2}}^{2}+\|u\|_{L^{2}}+\|v\|_{L^{2}}^{2}\right)\left\|v_{m}-v\right\|_{L^{2}}^{2},
\end{aligned}
$$

which tends 0 as $m \rightarrow \infty$.

By relations (2.12)-(2.17) we conclude that $\left\{w_{m}\right\}$ converges strongly to $w$ in $H$. Thus, the functional $T_{\lambda}$ satisfies the Palais-Smale condition in $H$.

Proof of Theorem 1.3. By Lemmas 2.1-2.4, for all $\lambda \geq \lambda^{*}$, system (1.1) admits a non-negative, non-trivial weak solution $w_{1}$ as the global minimizer of $T_{\lambda}$. Setting

$$
\bar{c}:=\inf _{\gamma \in \boldsymbol{\Gamma}} \max _{w \in \gamma([0,1])} T_{\lambda}(w)
$$

where

$$
\boldsymbol{\Gamma}:=\left\{\gamma \in C([0,1], H): \gamma(0)=0, \gamma(1)=w_{1}\right\} .
$$

Lemmas 2.5-2.6 show that all assumptions of the mountain pass theorem in [1] are satisfied, $T_{\lambda}\left(w_{1}\right)<0$ and $\left\|w_{1}\right\|>\rho$. Then, $\bar{c}$ is a critical value of $T_{\lambda}$, i.e., there exists $w_{2} \in H$ such that $T_{\lambda}^{\prime}\left(w_{2}\right)(\varphi)=0$ for all $\varphi \in H$ or $w_{2}$ is a weak solution of (1.1). Moreover, $w_{2}$ is a not trivial solution and $w_{2} \not \equiv w_{1}$ since $T_{\lambda}\left(w_{2}\right)=\bar{c}>0>T_{\lambda}\left(w_{1}\right)$. Theorem 1.3 is completely proved.

\section{Final comments}

In this section, we make some comments regarding extensions of system (1.1). While uniform elliptic problems (equations and systems) are intensively studied in the last decades, the degenerate elliptic problems still contain some unknown things, especially problems involving Grushin-type operators.

Firstly, the operator $G_{s}=\Delta_{x}+|x|^{2 s} \Delta_{y}$ can be naturally extended to the more complicated form

$$
\Delta_{x_{0}}+\sum_{i=1}^{m}\left|x_{0}\right|^{2 s_{i}} \Delta_{x_{i}}
$$

in the domain $\Omega=\left\{\left(x_{0}, x_{1}, \ldots, x_{m}\right): x_{i} \in \mathbb{R}^{N_{i}}, i=0,1, \ldots, m\right\}$. Then system (1.1) becomes

$$
\left\{\begin{aligned}
\Delta_{x_{0}} u+\sum_{i=1}^{m}\left|x_{0}\right|^{2 s_{i}} \Delta_{x_{i}} u & =\lambda F_{u}\left(x_{0}, x_{1}, \ldots, x_{m}, u, v\right) & & \text { in } \Omega \\
\Delta_{x_{0}} v+\sum_{i=1}^{m}\left|x_{0}\right|^{2 s_{i}} \Delta_{x_{i}} v & =\lambda F_{v}\left(x_{0}, x_{1}, \ldots, x_{m}, u, v\right) & & \text { in } \Omega \\
u=v & =0 & & \text { on } \partial \Omega
\end{aligned}\right.
$$


where $\Omega \subset \mathbb{R}^{N}=\mathbb{R}^{N_{1}} \times \mathbb{R}^{N_{2}}$ is a bounded domain with smooth boundary $\partial \Omega$, $0 \in \Omega$, and $\lambda$ is a positive parameter. Following [5, 22], the critical exponent for this case is

$$
\frac{N_{0}+\sum_{i=1}^{m}\left(s_{i}+1\right) N_{i}+2}{N_{0}+\sum_{i=1}^{m}\left(s_{i}+1\right) N_{i}-2} .
$$

In [5], the authors studied the existence of a nontrivial solution for (3.19) in the superlinear case. More precisely, the nonlinear term was assumed to satisfy the Ambrosetti-Rabinowitz type condition in [1], see the condition (H2) of [5].

In this work, we do not use this condition. Putting $x=\left(x_{0}, x_{1}, \ldots, x_{m}\right)$, we can preserve the hypotheses (f1)-(f4) and proceed with the functional energy

$$
\begin{aligned}
T_{\lambda}(w)= & \frac{1}{2} \int_{\Omega}\left(\left|\nabla_{x_{0}} u\right|^{2}+\sum_{i=1}^{m}\left|x_{0}\right|^{2 s_{i}}\left|\nabla_{x_{i}} u\right|^{2}\right) d x \\
& +\frac{1}{2} \int_{\Omega}\left(\left|\nabla_{x_{0}} v\right|^{2}+\sum_{i=1}^{m}\left|x_{0}\right|^{2 s_{i}}\left|\nabla_{x_{i}} v\right|^{2}\right) d x-\lambda \int_{\Omega} F(x, u, v) d x .
\end{aligned}
$$

Secondly, it should be noticed that using the same argument used above, we can deal with the system of $m$ unknowns

$$
\left\{\begin{aligned}
L_{\alpha_{1}, \alpha_{2}, \ldots, \alpha_{m} w} & =\lambda \nabla F & & \text { in } \Omega, \\
w & =0 & & \text { on } \partial \Omega,
\end{aligned}\right.
$$

where $w=\left(u_{1}, u_{2}, \ldots, u_{m}\right)$

$L_{\alpha_{1}, \alpha_{2}, \ldots, \alpha_{m}}=\left(\begin{array}{cccc}-G_{\alpha_{1}} & 0 & & 0 \\ 0 & -G_{\alpha_{2}} & \ldots & 0 \\ 0 & 0 & & -G_{\alpha_{m}}\end{array}\right), G_{s}=\Delta_{x}+|x|^{2 s} \Delta_{y}$ for $s \geq 0$,

and $\nabla F=\left(F_{u_{1}}, F_{u_{2}}, \ldots, F_{u_{m}}\right)$ stands for the gradient of a $C^{1}$ function $F$ : $\Omega \times \mathbb{R}^{m} \rightarrow \mathbb{R}$ which may change sign, $\Delta_{x}=\sum_{i=1}^{N_{1}} \frac{\partial^{2}}{\partial x_{i}^{2}}$, and $\Delta_{y}=\sum_{j=1}^{N_{2}} \frac{\partial^{2}}{\partial y_{j}^{2}}$. More precisely, we assume that $F: \Omega \times \mathbb{R}^{m} \rightarrow \mathbb{R}$ is a $C^{1}$-function, satisfying the following conditions:

$(\mathbf{f 1})^{\prime} F(x, y, 0,0, \ldots, 0)=0$ for a.e. $(x, y) \in \Omega$, and $F\left(x, y, t_{1}, \ldots, t_{i-1}, t_{i}\right.$, $\left.t_{i+1}, \ldots, t_{m}\right)=F\left(x, y, t_{1}, \ldots, t_{i-1}, 0, t_{i+1}, \ldots, t_{m}\right)$ for all $t_{i} \leq 0, t_{j} \in \mathbb{R}$ for all $j \neq i$ and a.e. $(x, y) \in \Omega, i, j=1,2, \ldots, m$;

$(\mathbf{f 2})^{\prime}$ There exists a constant $C>0$ such that

$$
\sum_{i=1}^{m}\left|F_{t_{i}}\left(x, y, t_{1}, t_{2}, \ldots, t_{m}\right)\right| \leq C\left(1+\sum_{i=1}^{m}\left|t_{i}\right|\right)
$$

for all $t=\left(t_{1}, t_{2}, \ldots, t_{m}\right) \in \mathbb{R}^{m}$ and a.e. $(x, y) \in \Omega$.

We say that a function $\gamma$ verifies the property $(\Gamma)^{\prime}$ if and only if

$$
\gamma\left(t_{1}, t_{2}, \ldots, t_{m}\right) \leq M \sum_{i=1}^{m}\left|t_{i}\right|^{2}
$$


for all $t=\left(t_{1}, t_{2}, \ldots, t_{m}\right) \in \mathbb{R}^{m}$, where $M>0$ is independent of $\gamma$.

Let $H_{i}, i=1,2$ be two functions satisfying property $\Gamma^{\prime}$ and denote

$$
|t|_{\mathbb{R}^{m}}=\left(\sum_{i=1}^{m}\left|t_{i}\right|^{2}\right)^{\frac{1}{2}}, \quad t=\left(t_{1}, t_{2}, \ldots, t_{m}\right) \in \mathbb{R}^{m} .
$$

We introduce the following assumptions on the behavior of $F$ at origin and at infinity:

$(\mathbf{f} 3)^{\prime}$ It holds that

$$
\limsup _{|t|_{\mathbb{R}^{m}} \rightarrow 0} \frac{F\left(x, y, t_{1}, t_{2}, \ldots, t_{m}\right)}{H_{1}\left(t_{1}, t_{2}, \ldots, t_{m}\right)} \leq 0
$$

uniformly in $(x, y) \in \Omega$;

$(\mathbf{f} 4)^{\prime}$ It holds that

$$
\limsup _{|t|_{\mathbb{R}^{m}} \rightarrow \infty} \frac{F\left(x, y, t_{1}, t_{2}, \ldots, t_{m}\right)}{H_{2}\left(t_{1}, t_{2}, \ldots, t_{m}\right)} \leq 0
$$

uniformly in $(x, y) \in \Omega$.

Then, using the similar arguments as in the proofs of Theorems 1.2 and 1.3, we can obtain the following results.

Theorem 3.1. Assume $F: \Omega \times \mathbb{R}^{m} \rightarrow \mathbb{R}$, satisfies

$$
\sum_{i=1}^{m}\left|F_{t_{i}}\left(x, y, t_{1}, t_{2}, \ldots, t_{m}\right)\right| \leq C \sum_{i=1}^{m}\left|t_{i}\right|, \quad C>0
$$

for all $t=\left(t_{1}, t_{2}, \ldots, t_{m}\right) \in \mathbb{R}^{m}$ and a.e. $(x, y) \in \Omega$. Then there is a constant $\lambda_{*}>0$ such that system (3.20) has no nontrivial weak solution for any $\lambda<\lambda_{*}$.

Theorem 3.2. Assume that the conditions $(\mathbf{f} \mathbf{1})^{\prime}-(\mathbf{f} \mathbf{4})^{\prime}$ are satisfied. Moreover, if in addition we assume that there exist a ball $B \subset \Omega$ and $t_{i}^{0}>0, i=1,2, \ldots, m$ such that $F\left(x, y, t_{1}^{0}, t_{2}^{0}, \ldots, t_{m}^{0}\right)>0$ for all $(x, y) \in B$, then there exists a constant $\lambda^{*}>0$ such that system (3.20) has at least two non-trivial, non-negative weak solutions for any $\lambda \geq \lambda^{*}$.

It is clear that our arguments in this paper are applicable to elliptic problems involving the Caffarelli-Kohn-Nirenberg inequalities in order to obtain better results in $[7,16,18]$. Finally, the study of existence of solutions for problem (1.1) with critical exponents is an interesting topic, see [21, 25].

Acknowledgements. The author would like to thank the referees for their suggestions and helpful comments which improved the presentation of the original manuscript. This work was supported by Vietnam National Foundation for Science and Technology Development (NAFOSTED). 


\section{References}

[1] A. Ambrosetti and P. H. Rabinowitz, Dual variational methods in critical points theory and applications, J. Funct. Anal. 4 (1973), 349-381.

[2] L. Boccardo and D. G. de Figueiredo, Some remarks on a system of quasilinear elliptic equations, Nonlinear Differential Equations Appl. 9 (2002), no. 3, 309-323.

[3] J. F. Bonder, Multiple positive solutions for quasilinear elliptic problems with signchanging nonlinearities, Abstr. Appl. Anal. 2004 (2004), no. 12, 1047-1056.

[4] N. M. Chuong and T. D. Ke, Existence of solutions for a nonlinear degenerate elliptic system, Electron. J. Differential Equations 2004 (2004), no. 93, 1-15.

[5] _ Existence results for a semilinear parametric problem with Grushin type operator, Electron. J. Differential Equations 2005 (2005), no. 107, 1-12.

[6] F. Catrina and Z. Q. Wang, On the Caffarelli-Kohn-Nirenberg inequalities: sharp constants, existstence (and non existstence) and symmetry of extremal functions, Comm. Pure Appl. Math. 54 (2001), no. 2, 229-258.

[7] N. T. Chung and H. Q. Toan, On a class of degenerate and singular elliptic systems in bounded domains, J. Math. Anal. Appl. 360 (2009), no. 2, 422-431.

[8] R. Dautray and J. L. Lions, Mathematical Analysis and Numerical Methods for Science and Technology I: Physical Origins and Classical Methods, Springer-Verlag, Berlin, 1985.

[9] V. Felli and M. Schneider, A note on regularity of solutions to degenerate elliptic equations of Caffarelli-Kohn-Nirenberg type, Adv. Nonlinear Stud. 3 (2003), no. 4, 431-443.

[10] V. V. Grushin, On a class of elliptic pseudo differential operators degenerate on a submanifold, Math. USSR Sbornik 13 (1971), 155-183.

[11] L. Iturriaga, Existence and multiplicity results for some quasilinear elliptic equation with weights, J. Math. Anal. Appl. 339 (2008), no. 2, 1084-1102.

[12] T. D. Ke, Existence of non-negative solutions for a semilinear degenerate elliptic system, Proceedings of the international conference on Abstract and Applied Analysis (Hanoi Aug. 2002), World Scientific, July 2004.

[13] T. T. Khanh and N. M. Tri, On the analyticity of solutions to semilinear differential equations degenerated on a submanifold, J. Differential Equations 249 (2010), no. 10, 2440-2475.

[14] A. Kristály and C. Varga, Multiple solutions for a degenerate elliptic equation involving sublinear terms at infinity, J. Math. Anal. Appl. 352 (2009), no. 1, 139-148.

[15] F. Lascialfari and D. Pardo, Compact embedding of a degenerate Sobolev space and existence of entire solutions to a semilinear equation for a Grushin-type operator, Rend. Sem. Mat. Univ. Padova 107 (2002), 139-152.

[16] M. Mihăilescu, Nonlinear eigenvalue problems for some degenerate elliptic operators on $\mathbb{R}^{N}$, Bull. Belg. Math. Soc. Simon Stevin 12 (2005), no. 3, 435-448.

[17] M. Mihăilescu, G. Morosanu, and D. S. Dumitru, Equations involving a variable exponent Grushin-type operator, Nonlinearity 24 (2011), no. 10, 2663-2680.

[18] M. Mihăilescu and V. Rădulescu, Ground state solutions of nonlinear singular Schrödinger equations with lack compactness, Math. Methods Appl. Sci. 26 (2003), no. 11, 897-906.

[19] M. K. V. Murthy and G. Stampachia, Boundary value problems for some degenerate elliptic operators, Ann. Mat. Pura Appl. 80 (1968), 1-122.

[20] K. Perera, Multiple positive solutions for a class of quasilinear elliptic boundary-value problems, Electron. J. Differential Equations 2003 (2003), no. 7, 1-5.

[21] V. Radulescu and D. Smets, Critical singular problems on infinite cones, Nonlinear Anal. 54 (2003), no. 6, 1153-1164.

[22] N. T. C. Thuy and N. M. Tri, Some existence and non-existence results for boundary value problems (BVP) for semilinear elliptic degenerate operators, Russ. J. Math. Phys. 9 (2002), no. 3, 366-371. 
[23] _ Nontrivial solutions to boundary value problems for semilinear strongly degenerate elliptic differential equations, Nonlinear Differential Equations Appl. 19 (2012), no. $3,279-298$.

[24] N. M. Tri, On Grushin's Equation, Matemachicheskie Zametki 63 (1988), 95-105.

[25] _ Critical Sobolev exponent for degenerate elliptic operators, Acta Math. Vietnamica 23 (1998), no. 1, 83-94.

[26] B. J. Xuan, The eigenvalue problem of a singular quasilinear elliptic equation, Electron. J. Differential Equations 2004 (2004), no. 16, 1-11.

Department of Mathematics and Informatics

QUANG Binh UNIVERSITY

312 Ly Thuong Kiet, Dong Hoi, Quang Binh, Vietnam

E-mail address: ntchung82@yahoo.com 